\title{
CRECIMIENTO ECONÓMICO, POLÍTICAS SOCIALES Y DISMINUCIÓN DE LA POBREZA
}

\section{ECONOMIC GROWTH, SOCIAL POLICY AND POVERTY REDUCTION}

\author{
Rey Navarro Walter
}

Facultad de Administración de Empresas de la Universidad Nacional del Centro del Perú.

\section{RESUMEN}

En la última década el Perú ha experimentado un crecimiento económico importante, sin embargo ha sido muy diferenciado entre sus departamentos, lo que merece especial atención pues el mismo es el mejor instrumento de lucha contra la pobreza. Sin embargo no sólo hay que tener en cuenta el crecimiento persé, sus características son importantes, pues a pesar del crecimiento, vastos sectores de la población podrían seguir siendo pobres. Si mediante las políticas públicas implementadas por el Estado se logran mayores coberturas y mejor distribución en el acceso a servicios básicos para la población, se tendrán mejores indicadores en nutrición, mortalidad infantil, años de instrucción, matriculación escolar, saneamiento, agua y electricidad; consecuentemente se incrementaran las capacidades de las personas, haciendo posible que la disminución de la pobreza será más sostenible en relación a las políticas que centren su accionar en el crecimiento económico y la mera disminución de la pobreza monetaria. La modificación de las tasas de pobreza e indigencia, según la CEPAL, se puede descomponer en dos: crecimiento del ingreso medio de las personas (efecto crecimiento) y las modificaciones en la forma en que se distribuye este ingreso (efecto distribución). Para el caso del Perú, la disminución de la pobreza entre el 2002 y el 2009, se ha debido en un 78\% al crecimiento y sólo en 22\% a la mejora en la distribución.

\section{SUMMARY}

In the last decade, Peru has experienced a significant economic growth, however, it has been highly differentiated among departments, which deserves special attention because it is the best tool for poverty fighting. But not only the growth must be taken into account perse, its characteristics are important because despite the growth, vast sectors of the population could remain poor. If by means of public policies implemented by the state is possible to achieve greater coverages and better distributionof accesses to basic services for the population, to obtain better nutrition indicators, infant mortality, education years, school enrollment, sanitation, water and electricity; capacity of people will be consequently increased, enabling poverty reduction to be more sustainable in relation to policies that focus their actions on economic growth and mere monetary poverty reduction. The change in poverty and extreme poverty rates, according to ECLAC, can be decomposed into two: people income growth (growth effect) and changes inthe way income is distributed (distribution effect). In the case of Peru, the declining in poverty between 2002 and 2009 was due to $78 \%$ growth and only $22 \%$ to the improvement in distribution. 


\section{INTRODUCCIÓN}

Como ya ha sido demostrado en extenso, el crecimiento económico es el mejor instrumento para disminuir la pobreza. Esta disminución de la pobreza vía crecimiento puede ser directa o indirecta, directamente mediante la generación de empleo y las mayores remuneraciones pagadas a los que ya tienen empleo; la vía indirecta es que el crecimiento económico permite la captación de más impuestos, los que hacen posible la implementación de las políticas sociales. Las políticas sociales permiten tener gente con más capacidades y será más fácil que en contextos de disminución del crecimiento puedan conseguir o generar su propio empleo.

Si mediante las políticas públicas implementadas por el Estado se logran mayores coberturas y mejor distribución en el acceso a servicios básicos para la población, se tendrán mejores indicadores en nutrición, mortalidad infantil, años de instrucción, matriculación escolar, saneamiento, agua y electricidad; consecuentemente se incrementaran las capacidades de las personas, haciendo posible que la disminución de la pobreza será más sostenible en relación a las políticas que centren su accionar en el crecimiento económico y la mera disminución de la pobreza monetaria.

Como se sustenta a lo largo de la investigación, sin embargo, la disminución de la pobreza por acción del Estado mediante el gasto público dependerá de la eficiencia, equidad, eficacia y transparencia de su gestión, aspectos en los cuales hay mucho por hacer.

Entonces, para disminuir no solo la pobreza monetaria sino también la no monetaria y lograr mayores niveles de desarrollo humano, la participación del Estado debe ser potenciada, es decir, desempeñar un rol más activo, pues como se ve, muchas de las variables para reducir los distintos tipos de pobreza implican necesariamente la intervención del Estado. Si no se tienen activas políticas sociales como en el caso del Perú, se explica por qué el "chorreo", producto del crecimiento económico, no ha beneficiado automáticamente ni beneficiará a vastos sectores de la población pobre; entonces. ¿Cómo conseguir un empleo con una remuneración adecuada a pesar del crecimiento económico si se tiene pocos años de educación y ésta es pésima?

Diversos estudios han puesto en evidencia que una mayor equidad impacta significativamente en el crecimiento económico, es decir que a menores desigualdades el crecimiento será mayor y a mayores niveles de desigualdad sucede lo contrario. Si una sociedad mediante políticas sociales activas; por ejemplo, logra mayores coberturas y también mejor distribución de la dotación de servicios tales como salud y educación, estará otorgando mayores oportunidades a la población pobre, excluida, permitiéndoles incrementar su productividad, incorporarse al mercado, percibir mayores remuneraciones y disminuir la desigual distribución de ingresos; aportando activamente al crecimiento económico.

Contrariamente, determinados segmentos de la población con mínimos niveles educativos y de pésima calidad, sin buena salud, tendrá poca productividad, consecuentemente su aporte a la producción nacional será menor comparativamente con aquellas que tienen buena educación y salud. Además si su salud es precaria se enfermará más y su esperanza de vida será menor, contribuyendo relativamente menos a la producción nacional. La información presentada en el Capítulo I, permite corroborar por qué sobre todo el área rural tiene enormes desventajas respecto al área urbana, o la sierra frente a la costa respecto a educación y salud, por ejemplo, para beneficiarse del crecimiento económico.

En la última década América Latina y el Caribe ha experimentado niveles importantes de crecimiento económico, lo que ha permitido financiar más gasto social, pero esto no basta, la calidad del gasto social es trascendente. La baja calidad del gasto, caracterizada por su ineficiencia, ineficacia, inequidad y la poca transparencia juegan en contra de los logros sociales, es decir que aunque se destinen más recursos al gasto social, los avances en los indicadores serán magros debido a la poca calidad del gasto, tal como sucede en el Perú, cuyos indicadores sociales dejan mucho que desear.

\section{MATERIAL Y MÉTODOS: \\ Material}

En el desarrollo del presente trabajo de investigación se han recurrido al uso de materiales, como material bibliográfico, informes, textos, así como material de escritorio, computadora, plumones, lapiceros, papeles, calculadoras, cuestionario, etc. 


\section{MÉTODOS}

\section{Método general: sistémico}

Sistema es un conjunto organizado de cosas o partes interactuantes e interdependientes, que se relacionan formando un todo complejo, identificable y distinto. Las cosas o partes que componen al sistema, no se refieren al campo físico (objetos), sino más bien al aspecto funcional. El método sistémico está dirigido a modelar el objeto mediante la determinación de sus componentes, así como las relaciones entre ellos.

\section{Método específico: Inductivo - Deductivo}

Para los proponentes de este esquema la ciencia se inicia con observaciones individuales, a partir de las cuales se plantean generalizaciones cuyo contenido rebasa el de los hechos inicialmente observados. Las generalizaciones permiten hacer predicciones cuya confirmación las refuerza y cuyo fracaso las debilita y puede obligar a modificarlas 0 hasta rechazarlas. El método inductivo-deductivo acepta la existencia de una realidad externa y postula la capacidad del hombre para percibirla a través de sus sentidos y entenderla por medio de su inteligencia; para muchos partidarios de este esquema, también nos permite explotarla en nuestro beneficio ${ }^{1}$.

\section{RESULTADOS Y DISCUSIÓN:}

En el presente capítulo se realiza el análisis e interpretación de los resultados de la investigación.

\section{Resultados}

Como ya ha sido demostrado el crecimiento económico es el mejor instrumento de la lucha contra la pobreza, pero las políticas sociales también son muy importantes. En el caso del Perú por sus indicadores sociales, como por los recursos destinados al gasto social, y por la calidad de éste se puede concluir que no es un país caracterizado por tener activas políticas sociales, las que han demostrado ser muy importantes para el crecimiento económico, la disminución de la pobreza y las desigualdades y lograr mejores indicadores de desarrollo humano. Relativos altos logros sociales y baja pobreza se dan en los casos donde se ha tenido una larga tradición de políticas 1 Pablo Rico Gallegos "El Método Científico" sociales, tales como en el caso de Chile y Costa Rica. Contrariamente en casos de ausencia de una larga tradición de políticas sociales y en contextos de rápido crecimiento, no se experimentan más rápido desarrollo social o disminución de la pobreza, casos como el de Bolivia y Paraguay. Uruguay muestra un relativo alto desarrollo social y baja pobreza en un contexto de lento crecimiento pero de políticas sociales activas y de larga data (Olavarría, 2005, pág. 8).

Cabe destacar que políticas sociales activas y de larga tradición permiten disminuir las desigualdades y de esta manera disminuir la pobreza, incrementar el crecimiento económico y lograr mayor desarrollo humano. Existe una evidente relación negativa entre desigualdad y desarrollo humano. La desigualdad en salud, educación e ingresos incide negativamente en el IDH, lo que es más acentuado en el caso de la educación e ingresos; por lo tanto se logrará mayor desarrollo humano si disminuye la desigualdad (PNUD, 2010, págs. 64-65). El crecimiento económico experimentado en la última década ha permitido incrementar significativamente el presupuesto público pero relajando los esfuerzos para incrementar la presión tributaria lo que no se debe repetir, dado el rol preponderante que tiene el Estado para un mayor crecimiento económico y desarrollo humano. Sin embargo, como también se ha señalado reiteradamente es muy importante a la vez mejorar la eficiencia, la equidad, la eficacia y la transparencia del gasto público; es decir mejorar sustancialmente su calidad. Pero para que un país tenga activas políticas sociales y se constituya en el otro pilar del desarrollo, la sociedad civil debe desarrollar un rol activo, para lo cual debe incrementar su capacidad de agencia, de reclamo, pues sin ella es muy poco probable que los encargados de tomar decisiones en el sector público adopten las medidas pro crecimiento y desarrollo sin exclusión. Una de las justificaciones que se pueden esgrimir para no haber logrado importantes avances en logros sociales y desarrollo humano, es el relativo poco crecimiento económico y la consecuente limitación de los recursos del Estado. Las experiencias han demostrado que varios países han logrado avanzar en el desarrollo humano a pesar de que sus economías no han crecido de manera significativa. Esto pondría en evidencia una mayor eficiencia, equidad y eficacia de las políticas públicas implementadas. 
Rebeca Arias, refiriéndose al Informe sobre el Desarrollo Humano 2010, señala que éste evidencia que existe poca correlación entre crecimiento económico y mejoras en salud o educación, pues en algunos países se produjeron mejoras en salud y educación, a pesar de un bajo crecimiento económico (El Comercio, 2010, pág. b7). Consideramos que los logros de estos países se debería a la mayor calidad del gasto público, lo que requiere un buen diseño e implementación de políticas públicas.

En suma, no sólo se trata de tener más recursos fiscales, es muy importante las políticas públicas y su implementación, la calidad del gasto público. Como señala AmartyaSen, se puede aumentar bastante la calidad de vida, aún si la renta son bajas, mediante un buen programa de servicios sociales. Dice que, dado que la educación y la salud contribuyen asimismo a acelerar el crecimiento económico, se justifica aún más el énfasis en los programas sociales en las economías pobres, sin esperar primero "hacerse rico" (2000, pág. 69).

Según Olavarría, en base al análisis comparativo que realizó a Chile, Uruguay, Costa Rica y Paraguay se tendría que el crecimiento económico chileno habría encontrado a vastos segmentos de la población saludables y educados, permitiéndoles aprovechar las oportunidades del crecimiento económico y salir de la pobreza. Afirma asimismo, en base al análisis comparativo realizado que un rápido crecimiento y relativamente bajos niveles de pobreza se dan en los casos en los que se tienen comparativamente buenos indicadores educativos y de salud, casos en los que se ha tenido largas y activas políticas sociales; por el contrario, el rápido crecimiento no conlleva a mayor desarrollo social o reducción de la pobreza en los casos de pocos logros sociales. Por tanto, el crecimiento económico y las políticas sociales son complementarias e interdependientes para reducir la pobreza (2005, pág. 9). En el caso peruano, si bien ha sido una de las economías que más ha crecido en la región, los logros sociales dejan mucho que desear, lo que pone en evidencia la ineficiencia e inequidad e ineficacia del gasto público social. Al respecto se han realizado diversos estudios (como el caso del Programa del Vaso de Leche), donde los mismos han demostrado que existe subcobertura y filtración, siendo utilizado como un programa clientelista, lo que limita la transparencia del mismo. Los resultados presentados en el capítulo I y la información que se presenta en el capítulo III sobre el despilfarro de recursos sustentan también lo afirmado.

Según el PNUD, dada una serie de estudios, sus resultados corroboran lo siguiente: el desarrollo humano no es igual que crecimiento económico y se pueden obtener grandes logros sociales a pesar de no tener crecimiento acelerado. Ello no significa dejar de lado el crecimiento, de lo que se trata es que a pesar de poco crecimiento se pueden enfrentar los retos en educación y salud; lo que es una buena noticia (2010, pág. 54). Esto es trascendente para la formulación e implementación de las políticas sociales pues normalmente se ha adoptado la actitud de que para hacer más y mejor en educación y salud se necesitan más recursos, pretextos que normalmente justifican la ineficiencia, inequidad e ineficacia del sector público caracterizado por una gestión burocrática - tradicional, donde a diferencia de la gestión por resultados los indicadores de desempeño tales como la eficiencia y la eficacia no cuentan. Esto no significa, para nada, que en el Perú no se necesite destinar muchos más recursos presupuestales a las políticas sociales, por supuesto que se necesita, pero poco se logrará si se siguen despilfarrando los recursos; se necesita entonces dar el gran salto de pasar de la gestión burocrática - tradicional a la gestión por resultados: por supuesto, esto será producto del rol activo de la sociedad civil, de su exigencia en el contexto de una profunda transformación del Estado.

Entonces las políticas sociales activas y sostenibles no serán producto solo de los que toman decisiones en el sector público, serán producto de una corriente de opinión que debe ser promovida por la sociedad civil en todo el país, donde los que pertenecen a las zonas rurales, excluidas, deben tener mayor participación, nos referimos a la capacidad de agencia de los ciudadanos en general. También es importante la participación de los formadores de opinión: líderes, dirigentes, políticos, etc. Particular importancia tienen los medios de comunicación, dejando de lado al periodismo barato que hacen algunos y que está caracterizado por la venta diaria de chismes, infundios, etc., u otros medios de comunicación que se dedican a ensalzar los aparentes logros de un gobierno, lo que por supuesto no es gratuito; contribuyendo a la desinformación, al 
desconocimiento de la realidad, tras lo cual muchas veces están oscuros intereses.

\section{DISCUSIÓN}

Como ya ha sido demostrado en extenso, el crecimiento económico es el mejor instrumento para disminuir la pobreza. Esta disminución de la pobreza vía crecimiento puede ser directa o indirecta. Aquí es importante hacer una precisión. Un escenario es el que se puede optar por priorizar la actuación del mercado para que la pobreza disminuya y no poner énfasis en las políticas sociales. Como consecuencia de ello; al producirse decrecimiento económico habrá trabajadores que fácilmente se quedarán sin empleo. Si en cambio, en el otro escenario, se hace de las políticas sociales una prioridad, se tendrá gente con más capacidades, de esta forma se podrán enfrentar mejor las crisis.

En otros términos, si la actividad económica se contrae es más fácil que se caiga por debajo de la línea de pobreza, según el enfoque de la pobreza monetaria si no se han implementado políticas sociales activas que permitan más y mejor salud y educación por ejemplo. Es decir, el impacto del decrecimiento es directo, se tendrá más desempleo y se generará más pobreza. Implementar políticas públicas activas, obteniendo mejores indicadores sobre todo en salud y educación, permite disminuir la pobreza monetaria y no monetaria y consecuentemente lograr mayor desarrollo humano.

Por tanto, comparativamente, si un país tiene políticas sociales activas de larga data, lo que le permite tener buenos indicadores sociales, las recesiones económicas generarán menos pobreza relativa respecto a políticas públicas que han priorizado fundamentalmente el crecimiento económico para disminuir la pobreza, dejando en un segundo plano las políticas sociales.

Si mediante las políticas públicas implementadas por el Estado se logran mayores coberturas y mejor distribución en el acceso a servicios básicos para la población, se tendrán mejores indicadores en nutrición, mortalidad infantil, años de instrucción, matrícula escolar, saneamiento, agua y electricidad; consecuentemente se incrementarán las capacidades de las personas, haciendo posible que la disminución de la pobreza sea más sostenible en relación a las políticas que centren su accionar en el crecimiento económico y la mera disminución de la pobreza monetaria.

Entonces, para disminuir no sólo la pobreza monetaria sino también la no monetaria y lograr mayores niveles de desarrollo humano, la participación del Estado debe ser potenciada, es decir, desempeñar un rol más activo, pues como se ve, muchas de las variables para reducir los distintos tipos de pobreza implican necesariamente la intervención del Estado; pero como se sostiene en el transcurso del ensayo, se requiere un Estado de calidad. Si no se tienen políticas sociales activas como en el caso del Perú, se explica por qué el "chorreo", producto del crecimiento económico, no ha beneficiado automáticamente ni beneficiará a vastos sectores de la población pobre; entonces por ejemplo ¿cómo conseguir empleo con una remuneración adecuada a pesar del crecimiento económico si se tiene pocos años de educación y ésta es pésima?

En resumen, el crecimiento económico alto pero sostenido y las políticas sociales activas son indispensables para disminuir la pobreza, incrementar el crecimiento y disminuir la desigualdad. Si se tienen mejores indicadores de salud y educación, así como en agua, saneamiento y electricidad, entre otros, las probabilidades de ser nuevamente pobres serán menores. Mejores indicadores económicos y sociales, permitirán incrementar la capacidad de agencia de la sociedad civil, lo que hará posible lograr un Estado de mejor calidad y el desarrollo de más y mejor mercado, lo que se traducirá en mayor crecimiento económico y desarrollo.

Diversos estudios han puesto en evidencia que una mayor equidad impacta significativamente en el crecimiento económico, es decir que a menores desigualdades el crecimiento será mayor. Si una sociedad mediante políticas sociales activas; por ejemplo, logra mayores coberturas y también mejor distribución de la dotación de servicios tales como salud y educación, estará otorgando mayores oportunidades a la población pobre, excluida, permitiéndoles incrementar su productividad, incorporarse al mercado, percibir mayores remuneraciones y disminuir la desigual distribución de ingresos; aportando activamente al crecimiento económico.

Contrariamente, determinados segmentos de la 
población con mínimos niveles educativos y de pésima calidad, sin buena salud, tendrá poca productividad, consecuentemente su aporte a la producción nacional será menor comparativamente con aquellas que tienen buena educación y salud. Además si su salud es precaria se enfermará más y su esperanza de vida será menor, contribuyendo relativamente menos a la producción nacional. La información presentada en el Capítulo I, permite corroborar por qué sobre todo el área rural tiene enormes desventajas respecto al área urbana, o la sierra y la selva frente a la costa respecto a educación y salud, por ejemplo, para beneficiarse del crecimiento económico.

De acuerdo al Banco Mundial la pobreza puede crear un círculo vicioso: bajo crecimiento económico genera altos índices de pobreza, esto a la vez genera poco crecimiento económico. Este círculo vicioso es generado, entre otros, por la falta de acceso a salud, a educación y a infraestructura de calidad, debido a las malas políticas públicas (Banco Mundial, 2011, pág. 11). Efectivamente, cómo se pueden esperar buenas políticas públicas si el Estado en muchos casos es un botín, copado en unos gobiernos más que en otros, por gente sin ningún tipo de calificación, refugio de candidatos que no fueron elegidos como alcaldes o regidores, pero que como el candidato de su agrupación política ganó, tienen el "derecho", según ellos, de ocupar un puesto público.

Pero la equidad, la justicia, no nos debe preocupar sólo porque es un instrumento para lograr mayores niveles de crecimiento económico (mediante el incremento de la productividad), que sería el enfoque del capital humano, interesa la equidad por ser instrumento y finalidad de sociedades desarrolladas. Precisamente las sociedades de este tipo se caracterizan por una menor desigualdad en las dimensiones del desarrollo (salud, educación e ingresos) así como menor desigualdad territorial, entre otros.

El crecimiento económico es muy importante para reducir la pobreza pero no es suficiente, las activas políticas sociales del Estado son insustituibles para reducir la pobreza tanto monetaria y no monetaria y para lograr mayores niveles de desarrollo humano. Según el PNUD bajo el enfoque del desarrollo humano se concibe a los seres humanos como beneficiarios y agentes promotores del desarrollo, concibiendo el desarrollo humano como la expansión de las libertades de las personas para tener una larga vida, saludable y creativa, lograr metas que consideren valiosas e intervenir para tener un desarrollo equitativo y sostenible (INFORME SOBRE DESARROLLO HUMANO 2010. Edición del Vigésimo Aniversario., 2010, pág. 24).

Sin embargo, el Perú no es precisamente una economía caracterizada por activas políticas sociales y los indicadores; por ejemplo, de salud y de educación, así lo ponen en evidencia, convirtiéndose en una severa restricción para disminuir la

pobreza, promover el crecimiento económico y lograr mayor desarrollo humano. A pesar de haber experimentado uno de los mayores niveles de crecimiento económico acumulado en la década pasada a nivel de América Latina y el Caribe, exhibe magros indicadores sociales en la región, caracterizándose además por ser uno de los países con más desigualdad, tal como se evidencia con la información presentada.

\section{CONCLUSIONES}

- El crecimiento económico es el mejor instrumento de la lucha contra la pobreza, pero las políticas sociales también son muy importantes. En el caso del Perú por sus indicadores sociales, como por los recursos destinados al gasto social, y por la calidad de éste se puede concluir que no es un país caracterizado por tener activas políticas sociales, las que han demostrado ser muy importantes para el crecimiento económico, la disminución de la pobreza y las desigualdades y lograr mejores indicadores de desarrollo humano.

- Las políticas sociales activas y de larga tradición permiten disminuir las desigualdades y de esta manera disminuir la pobreza, incrementar el crecimiento económico y lograr mayor desarrollo humano.

- Una de las justificaciones que se pueden esgrimir para no haber logrado importantes avances en logros sociales y desarrollo humano, es el relativo poco crecimiento económico y la consecuente limitación de los recursos del Estado. Las experiencias han demostrado que varios países han logrado avanzar en el desarrollo humano a pesar de que sus economías 
no han crecido de manera significativa. Esto pondría en evidencia una mayor eficiencia, equidad y eficacia de las políticas públicas implementadas.

- Las políticas sociales activas y sostenibles no serán producto solo de los que toman decisiones en el sector público, serán producto de una corriente de opinión que debe ser promovida por la sociedad civil en todo el país, donde los que pertenecen a las zonas rurales, excluidas, deben tener mayor participación, nos referimos a la capacidad de agencia de los ciudadanos en general. También es importante la participación de los formadores de opinión: líderes, dirigentes, políticos, etc. Particular importancia tienen los medios de comunicación.

\section{REFERENCIAS BIBLIOGRÁFICAS}

- Banco Mundial. (2011). Perú en el umbral de una nueva era. (Vol. I). Washington, D.C.: Banco Mundial.

- CEPAL. (2010). Panorama Social de América Latina 2010. Santiago de Chile: Comisión Económica para América Latina y el Caribe.
- CEPLAN. (2011). Plan Bicentenario: El Perú hacia el 2021. Lima: Centro Nacional de Planeamiento Estratégico.

- PNUD. (2010). INFORME SOBRE DESARROLLO HUMANO 2010. Edición del Vigésimo Aniversario. EE.UU.: Programa de las Naciones Unidas para el Desarrollo.

- Olavarría M. (2005). Superación de la pobreza: ¿Crecimiento económico Vs Políticas sociales? X Congreso Internacional del CLAD sobre la Reforma del Estado y de la Administración Publica. Santiago de Chile

- Pablo Rico Gallegos(10 de marzo de 2013)" El Método Científico ". Obtenido de Foro de trabajos de Pablo Rico Gallegos:, Tema diario.:

http://psicopsi.com/EL_METODO_CIENTIFICO.asp 